\title{
Bridging Reality and Virtuality in Vocational Training
}

\author{
Kai Schmudlach, Eva Hornecker, Hauke Ernst, F. Wilhelm Bruns \\ Forschungszentrum Arbeit-Umwelt-Technik (artec) \\ University of Bremen, P. O. Box 330440, \\ 28334 Bremen, Germany \\ ++494212187307 \\ sks/eva/ernst/bruns@artec.uni-bremen.de
}

\begin{abstract}
This article describes work and results of BREVIE, a project aimed at designing and evaluating a new kind of environment for vocational training in pneumatics. It is based on the concept of graspable interfaces, which allow synchronous modelling in real and virtual worlds.
\end{abstract}

\section{Keywords}

Augmented Reality, Graspable User Interfaces, Tangible Interfaces, CSCW, Vocational Training, Pneumatics

\section{INTRODUCTION}

BREVIE (Bridging Reality and Virtuality with a Graspable User Interface) is a European sponsored project. It aims at designing, developing and evaluating a new kind of learning environment for vocational training in pneumatics. Pneumatics is a field with high relevance to industrial production. Approx. $80 \%$ of the producing factories employ pneumatics, that is compressed air, to control and drive machines. One main benefit of pneumatics is the possibility of implementing logical operations and circuits, just as well as distributing force easily. So this topic remains central to vocational training in the industrial context. The main characteristic of the BREVIE learning environment is to provide automatic transitions and links between two worlds which are currently separated for students: the physical circuit, which can be grasped with hands, and the world of abstract symbols which can be viewed on paper or on a computer screen.

Goal of the BREVIE learning environment is to contributes to the following pedagogical objectives: 1) acquisition of basic knowledge in the field of pneumatics, 2) ability to build experimental physical/functional pneumatic circuits, 3) ability to build pneumatic circuits with a digital simulator and to make use of a simulator for experimenting with pneumatic parts and circuits, 4) ability to recognise symbols of pneumatic components, to read pneumatic circuits and to design pneumatic circuits with speci- fied functionality, 5) ability to distinguish between different models of pneumatic circuits, to draw relations between these different models and to review pneumatic circuits from different points of view, 6) train the ability to solve a given task collaboratively in a group of students.

\section{BREVIE}

In vocational training for pneumatics several kinds of learning material can be found. For our project consortium, the most compelling is the popular pneumatic construction kit of our partner Festo Didactic which can be used for building functional circuits. These circuits work with compressed air and allow a very close representation of real

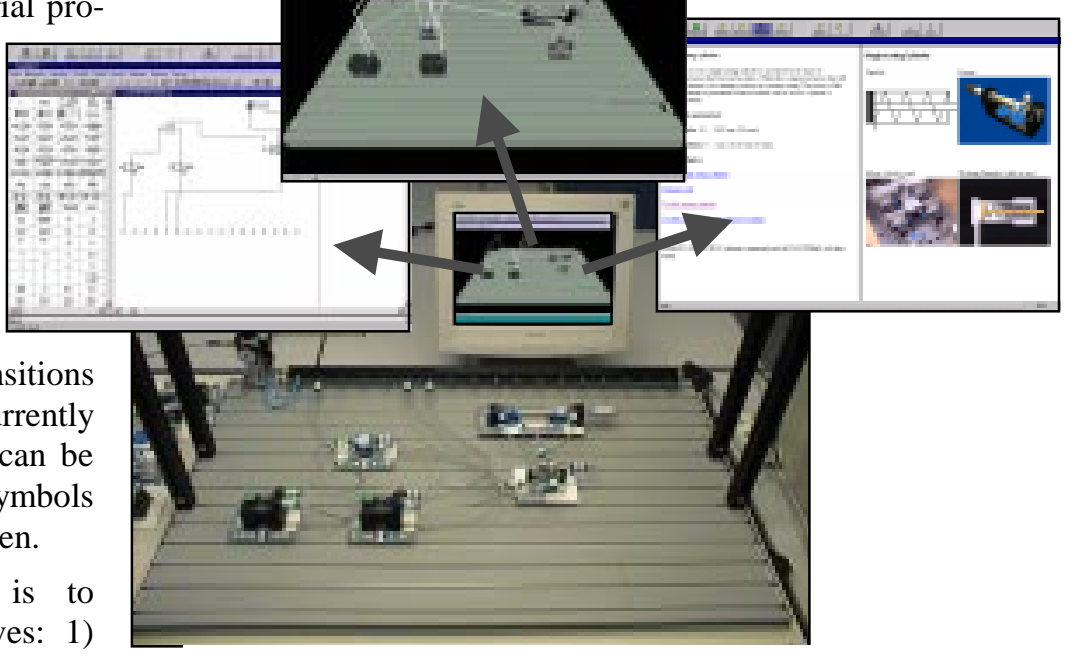

Figure 1 The BREVIE Learning Environment

production environments. BREVIE extends these construction kits by developing technical links to computer-based learning media. These automated links between real models and corresponding computer representations provide smooth transitions between multiple views on the same model to support the development of rich mental models.

We have integrated the following views, each with the possibility to interact with the model and to manipulate it: 1) the physical model, 2) the virtual model in 3D, 3) a symbolic/logical view in a simulation software for pneumatics 
In addition, the BREVIE Learning Environment provides access to multimedia learning material (hypertext, images, videos and diagrams) which introduces the functional properties of the pneumatic parts. The user can request this information right out of the physical modelling context with the help of a pointing device. Students just point onto the physical component with it to look up the component in the online database.

The link between computer based virtuality and reality is achieved by a "Universal Graspable User Interface" (short UGUI). The UGUI as an abstraction layer for different input device configurations is designed very modular, allowing to easily replace one kind of input-hardware by another, such as data-gloves (used in some of our other projects). In BREVIE we use two low cost video cameras which are mounted above the worktable and image recognition of colour bar-code labelled elements to synchronise the physical model with a virtual model (see Figure 2). This is the basis for further processing on the computer.

Since every application field requires a special combination of components we have introduced a highly modular system architecture which permits us to connect easily existing or new components. The design

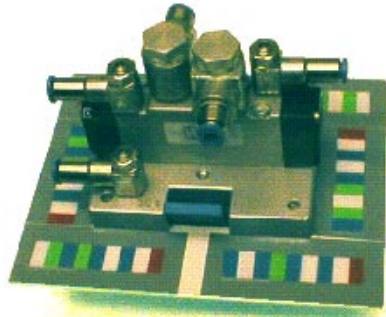

Figure 1: A Pneumatic Element with Colour Bar-Codes follows a client-server approach with a Real Object Manager (ROMAN) as the central server unit. Other software units - such as the UGUI, simulation tools, 3D viewers and hypertext browsers - are connected to ROMAN via tcp/ip based protocols.

BREVIE includes intensive evaluation by work psychologists from the Swiss Federal Institute of Technology Zurich, comparing learning processes and results with and without media support in four vocational schools all over Europe. The evaluation of the BREVIE system is divided into two phases: the first took place with an early prototype of the BREVIE system, the second is currently in progress with our new version. Evaluation followed a three-step (pre-test, process phase and post-test) procedure with three different learning environments (real FESTO components only, pneumatic simulator only and our integrated BREVIE learning environment) compared. These learning environments differ in the amount of tactile experience with pneumatics components, in the degree of abstraction and the combination of information formats. A 16 hours lesson plan was delivered to 89 students.

\section{Evaluation outcomes}

We measured the effects on learning output (knowledge in pneumatics and practical problem-solving). Despite of several technical problems and limited functionality of our prototype the BREVIE students gained a higher amount of pneumatics knowledge as all the other groups. Neverthe- less, the main factors for the learning output were previous knowledge in pneumatics and spatial abilities. The relevance of practical experience for fast problem-solving could be proofed. Based on the positive tendencies shown so far, we expect the results of the current evaluation to more clearly point out the advantages of our system.

In addition, it came out that advanced learners of pneumatics use the system in a different way than beginners: Beginners prefer to build up circuits with the hardware construction kit and use the online help system to lookup the functional properties of the pneumatic parts. Afterwards, they use the image recognition system to create the virtual model, validate it in the $3 \mathrm{D}$ view and recapitulate the circuit behaviour with the simulator. Advanced students usually start with the symbolic representation in the simulator, creating the circuit and simulating it. When they solved the task in the simulator, they use the automatically generated $3 \mathrm{D}$ view of the circuit as a pattern to build up the physical model.

Observations of school lessons, concentrating upon the role of graspable objects, enforced our belief in the BREVIE design. Working with real pneumatic elements was very motivating for students and led to curiosity regarding the internal functioning of components. When the teacher used computer tools not coupled with the real circuit, we observed that the computer tended to distract students. Thus computer tools must be tightly coupled with the task. The large pneumatic tables also seemed to invite collaboration, as nearby groups often watched their neighbours and helped out of their own accord.

\section{CONCLUSION}

Graspable interfaces as means for computer aided modelling allow using the same physical environment and foster cooperation and shared understanding. BREVIE will be introduced to the market as "CLEAR: The Constructive Learning Environment". It can be easily adapted to other application fields other than pneumatics.

\section{REFERENCES}

1 Bruns, F.W. Complex Construction Kits for Coupled Real and Virtual Engineering Workspaces. Streitz, N.A., Siegel, J., Hartkopf, V., Konomi, S. (eds) Proc. of Cooperative Buildings (CoBuild'99). Springer 1999. 55-68

2 Hornecker, E., Robben, B. Vocational Training with Combined Real/Virtual Environments. Proc. of HCI International 1999.

3 Ernst, H., Schäfer, K., Bruns, W. Creating Virtual Worlds with a Graspable User Interface. In Nijholt, A.; Donk, O.; Dijk, B. (Eds.) $15^{\text {th }}$ Twente Workshop on Language Technology (TWTL 15): Interactions in Virtual Worlds. University of Twente, NL, 1999 